\title{
Endothelium-dependent and independent vasodilation studied at normoglycaemia in Type I diabetes mellitus with and without microalbuminuria
}

\author{
G.Dogra ${ }^{1}$, L. Rich ${ }^{1}$, K.Stanton ${ }^{2}$, G.F. Watts ${ }^{1}$ \\ ${ }^{1}$ Department of Medicine and Western Australian Heart Research Institute, University of Western Australia, Perth, Australia \\ ${ }^{2}$ Department of Diabetes and Endocrinology, Royal Perth Hospital, Perth, Australia
}

\section{Abstract}

Aims/hypothesis. We examined whether endothelial function is impaired in patients with Type I (insulindependent) diabetes mellitus under conditions of near-normoglycaemia compared with age-matched healthy control subjects. Our aim was to determine whether microalbuminuria is associated with endothelial dysfunction in Type I diabetes.

Methods. Endothelial function, measured as postischaemic flow-mediated dilatation of the brachial artery using ultrasound, was compared among $17 \mathrm{mi}-$ croalbuminuric and 17 normoalbuminuric diabetic patients, and 17 control subjects. Glyceryl trinitratemediated dilatation of the brachial artery was used to measure endothelium-independent function. All diabetic patients were studied at near-normoglycaemia, using insulin and 5\% dextrose infusions to maintain blood glucose between 3.5 and $8.0 \mathrm{mmol} / 1$.

Results. Flow-mediated dilatation was significantly lower in microalbuminuric diabetic patients $(3.2 \pm 0.3 \%)$ compared with normoalbuminuric diabetic patients $(5.4 \pm 0.6 \%)$ and control subjects $(7.9 \pm 0.6 \%, p<0.001)$. Normoalbuminuric diabetic patients also had significantly lower flow-mediated dilatation than control subjects $(p=0.01)$. Glyceryl trinitrate mediated dilatation was significantly lower in the microalbuminuric patients compared with the control subjects $(11.9 \pm 1.1 \%$ vs $20.0 \pm 1.2 \%$, $p=0.001$ ). Albumin excretion rate and glycated haemoglobin showed a significant negative independent correlation with flow-mediated dilatation (both $p<0.05)$.

Conclusion/interpretation. Type I diabetic patients show endothelial dysfunction at near-normoglycaemia compared with the control subjects, and this abnormality is more marked in diabetic patients with microalbuminuria. Endothelial dysfunction in Type I diabetes is related to the albumin excretion rate and glycaemic control. The presence of endothelial dysfunction in normoalbuminuric diabetic patients suggests it could precede microalbuminuria as an early risk marker for cardiovascular disease. [Diabetologia (2001) 44: 593-601]

Keywords Endothelial dysfunction, Type I diabetes mellitus, microalbuminuria, hyperglycaemia, atherosclerosis.
Received: 6 November 2000 and in revised form: 11 January 2001

Corresponding author: Dr. G. Dogra, University Department of Medicine, Royal Perth Hospital, BOX X2213 GPO, Perth, Western Australia 6847

Abbreviations: ACR, albumin:creatinine ratio; ApoB, apolipoprotein B; DBP, diastolic blood pressure; DM, diabetes melli- tus; DMMA-, diabetes mellitus without microalbuminuria; DMMA +, diabetes mellitus with microalbuminuria; FMD, flow-mediated dilatation; GTN, glyceryl trinitrate; GTNMD, glyceryl trinitrate-mediated dilatation; HRT, hormone replacement therapy; Lp(a), lipoprotein (a); NO, nitric oxide; ROI, region of interest; SBP, systolic blood pressure; VWF, von Willebrand factor. 
Patients with Type I (insulin-dependent) diabetes mellitus and normoalbuminuria have a two- to fourfold increased risk of cardiovascular disease [1], increasing 20 to 40 times more in those with microalbuminuria $[1,2]$. Associated risk factors, such as smoking, hypertension and dyslipidaemia, only explain a small proportion of this increased cardiac risk [2]. Although modification of these conventional cardiac risk factors is essential in the management of patients with Type I diabetes, identification of new risk markers such as endothelial dysfunction, preferably before the onset of microalbuminuria, would allow earlier, more aggressive treatment of at risk patients.

Endothelial dysfunction is an early phase of atherosclerosis, associated mainly with an abnormality of the physiology of nitric oxide [3]. Endothelial dysfunction can be detected non-invasively using highresolution ultrasonography to measure post-ischaemic flow-mediated dilatation (FMD) of peripheral conduit arteries [4]. Conduit artery endothelial dysfunction is associated with abnormal vasomotor responses of the coronary circulation $[5,6]$ and conventional cardiac risk factors such as hypercholesterolaemia and smoking [4]. Endothelial dysfunction of the coronary and peripheral circulation has been shown to predict coronary events [7-9].

Although conduit artery studies support the presence of endothelial dysfunction in Type I diabetes $[10,11]$, particularly in microalbuminuric patients $[12,13]$, results are conflicting $[14,15]$. There are several possible reasons for the discrepancy in these findings. Firstly, acute hyperglycaemia impairs endothelial function in healthy and diabetic subjects [16, 17] and false positive results could be a consequence of varying ambient blood glucose concentrations at the time of the study. Secondly, methodological imprecision as a consequence of error associated with observer-dependent protocols for measuring the arterial diameter using ultrasonic callipers could contribute to the varied results. Thirdly, variation in longterm glycaemic control and degrees of diabetic complications among studies published could also contribute to the conflicting results. Finally, anti-hypertensive and, in particular angiotensin converting enzyme inhibitor (ACE-inhibitor) therapy, was not always uniform in studies of microalbuminuric patients.

We report on conduit artery endothelial function as measured by post-ischaemic FMD in patients with Type I diabetes with and without microalbuminuria, and in healthy control subjects matched for age and sex. Diabetic patients were studied under conditions of near-normoglycaemia and all microalbuminuric patients were on ACE-inhibitor therapy. Brachial artery changes were measured using a new computerised edge-detection algorithm which improved the precision of our measurements. We have also assessed endothelium-independent arterial responses to sub- lingual glyceryl trinitrate (GTN) and explored the association between vascular responses, microalbuminuria, lipid concentrations, disease duration, glycaemic control and in vivo markers of endothelial function.

\section{Subjects and methods}

Thirty-four patients aged 18 to 65 years, with Type I diabetes (C-peptide negative, ketosis prone) of at least 5 years duration, were recruited from the Diabetes Clinic at Royal Perth Hospital, Perth, Western Australia. Seventeen patients had persistent microalbuminuria, defined as a urinary albumin excretion rate of 20 to $200 \mu \mathrm{g} / \mathrm{min}$ in 2 out of 3 overnight urine collections over a period of 3 months and while on an ACE-inhibitor. All microalbuminuric diabetic patients were on an ACE-inhibitor for a minimum of 3 months before participating in the study. A further 17 diabetic patients without microalbuminuria and not on ACE-inhibitor therapy, and 17 healthy non-diabetic volunteers from the Perth metropolitan community were selected to match the microalbuminuric patients for age, sex and body mass index (BMI, $\left.\mathrm{kg} / \mathrm{m}^{2}\right)$. Subjects with untreated hypertension, clinically apparent cardiovascular disease, serum creatinine greater, than $120 \mu \mathrm{mol} / \mathrm{l}$ hypothyroidism, abnormal liver function, alcohol consumption of more than 3 standard drinks a day, primary lipid disorders and/or on lipidlowering therapy, drugs or agents known to influence endothelial function (ACE-inhibitor except in microalbuminuric patients, calcium channel blockers, aspirin, fish oils, vitamin and anti-oxidant preparations) and psychiatric disorders were excluded. The Ethics Committee at the Royal Perth Hospital approved the study and all subjects gave their written consent.

Study design and laboratory methods. The study was a crosssectional comparison of brachial artery vascular function among diabetic patients with and without microalbuminuria and healthy non-diabetic subjects. All subjects gave a detailed medical history and had a medical examination and electrocardiograph to assess inclusion and exclusion criteria. Blood pressure and heart rate were measured using a Dinamap 1846 SX/P monitor (Critikon, Tampa, Fla., USA) after resting the subjects for $10 \mathrm{~min}$ in the supine position. Venous blood samples were obtained after a 12-h fast, with minimal venous stasis and with the subjects in the supine position, for the following variables, measured by standard laboratory techniques unless stated otherwise: creatinine, albumin, plasma glucose, glycated haemoglobin $\left(\mathrm{HbA}_{1 \mathrm{c}}\right)$, total cholesterol, low-density lipoprotein (LDL) cholesterol (calculated using the modified Friedewald formula), high-density lipoprotein (HDL) cholesterol, triglycerides, vonWillebrand factor (vWF) and fibrinogen. Apolipoprotein B (apoB) and lipoprotein (a) $[\mathrm{Lp}(\mathrm{a})]$ were assayed by immunophelometric methods (Behring Nephelometer Analyzer II, Behring Diagnostics, NSW, Australia). Homocysteine was measured using a fluorescence polarisation immunoassay with the Abbott IMx Homocysteine kit B3D390 (Axis Biochemicals ASA, Ulvenveien 87, N-0581 Oslo, Norway) and the Abbott Imx analyser (Abbott, Australasia, NSW, Australia). Diabetic patients provided 3 overnight urine collections over 3 months for determination of microalbuminuria. On the study day, diabetic patients provided an overnight urine collection for assessment of albumin excretion rate (AER) and the urinary albumin to creatinine ratio (ACR). Control subjects provided a first morning urine sample to assess urinary ACR. The inter-assay coefficient of variation (CV) for all analytical methods was less than $6 \%$. 

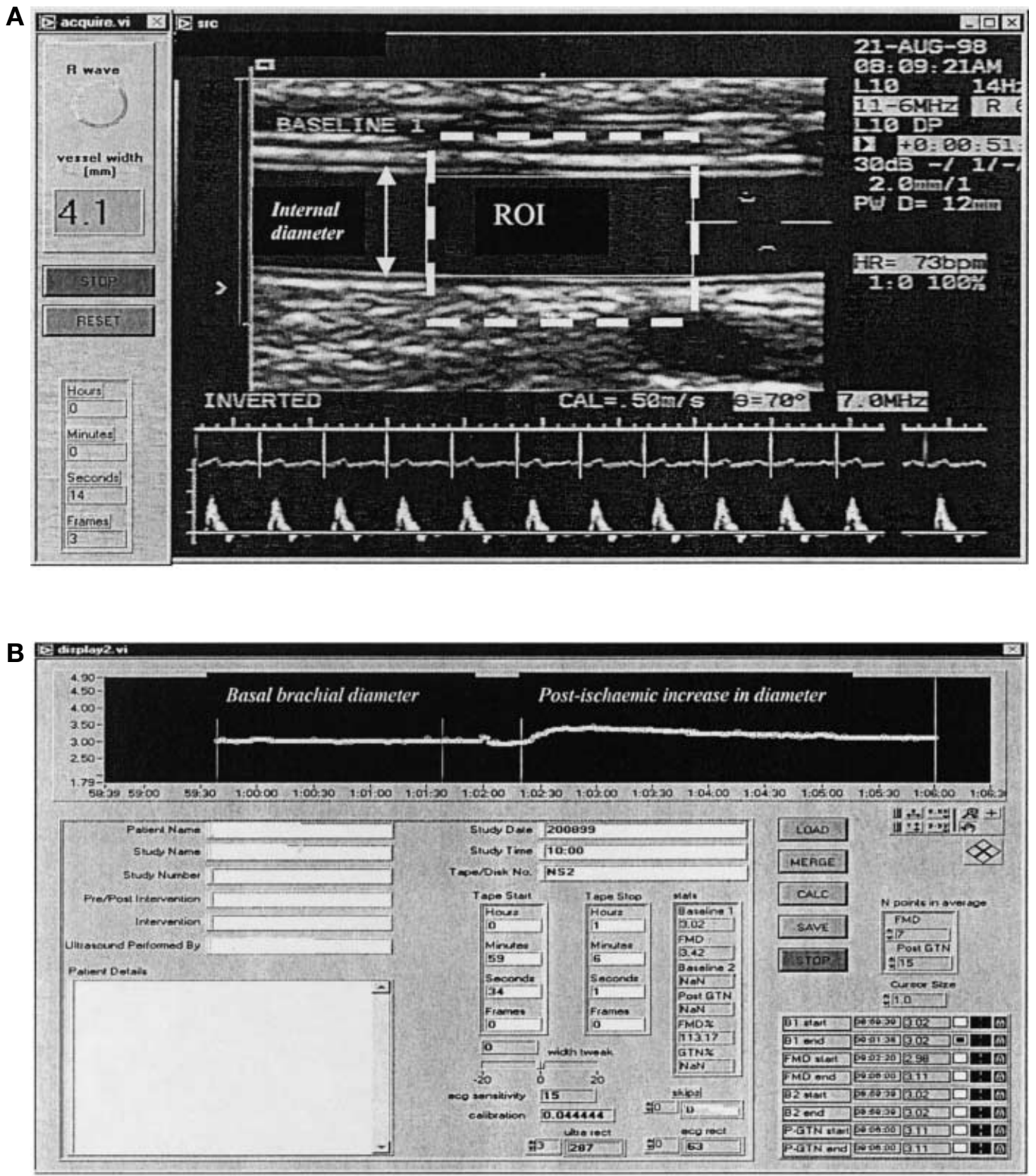

Fig. 1A, B. (A) Brachial artery ultrasound image acquisition and edge-detection by software system. (ROI - region of interest). (B) Basal and post-ischaemic brachial artery diameters as calculated by software system using a third-order polynomial.

Glomerular filtration rate (GFR) was calculated using the Cockcroft and Gault equation as follows: K · (140-age) - body weight in $\mathrm{kg} / \mathrm{serum}$ creatinine in $\mu \mathrm{mol} / \mathrm{l}$, with $\mathrm{K}$ equal to 1 for men and 0.85 for women.

Brachial artery ultrasonography. Brachial artery ultrasonography was carried out after a 12 -h fast from food and beverages, and after resting supine for at least $15 \mathrm{~min}$. Smokers were advised not to smoke on the day of the test. Before the ultrasound procedure, an 18-gauge cannula was inserted into an antecubital vein of the right forearm for insulin or $25 \%$ dextrose infusions, or both. Insulin and/or $25 \%$ dextrose was delivered as a continuous, constant-rate infusion over 60 to $90 \mathrm{~min}$ to achieve and then maintain blood glucose concentrations between 3.5 and $8 \mathrm{mmol} / \mathrm{l}$. Blood glucose concentrations were measured every $5 \mathrm{~min}$ until stable concentrations were maintained for at least $20 \mathrm{~min}$ before the ultrasound procedure. During the ultrasound, 3 blood glucose concentrations were measured at 10-min intervals: when beginning the ultrasound, after the post-ischaemic period and after GTN measurements were complete. The mean blood glucose during ultrasound was derived from these measurements.

For the ultrasound procedure, patients were rested supine in a quiet, temperature controlled room $\left(21-25^{\circ} \mathrm{C}\right)$. The left arm was immobilised in a foam cast and supported comfortably in extension. A 12-megahertz transducer connected to an Acuson Aspen 128 ultrasound device (Acuson Corporation, Mountainview, Calif., USA) was used for ultrasound. Continuous ECG monitoring was carried out in all studies. The trans- 
Table 1. Clinical characteristics of the diabetic patients with (DMMA + ) and without (DMMA-) microalbuminuria and the nondiabetic control subjects.

\begin{tabular}{|c|c|c|c|c|}
\hline & DM MA + & DM MA- & Controls subjects & $p$-value \\
\hline$n$ & 17 & 17 & 17 & \\
\hline Age (years) & $48.7(2.8)$ & $44.6(2.4)$ & $46.8(3.0)$ & 0.576 \\
\hline $\operatorname{Sex}($ men/women $)$ & $14 / 3$ & $10 / 7$ & $12 / 5$ & 0.322 \\
\hline Duration of diabetes (years) & $25.3(2.0)$ & $20.6(2.7)$ & - & 0.406 \\
\hline History of hypertension (yes/no) & $7 / 10^{\mathrm{a}}$ & $1 / 16$ & $0 / 17$ & 0.001 \\
\hline Retinopathy (yes/no) & $15 / 2$ & $9 / 8$ & - & 0.029 \\
\hline Smokers (yes/no) & $2 / 15$ & $3 / 14$ & $3 / 14$ & 0.862 \\
\hline HRT (yes/no) & $2 / 15$ & $3 / 10$ & $3 / 14$ & 0.206 \\
\hline Insulin dose (units/day) & $58(5.9)$ & $51(5.2)$ & - & 0.704 \\
\hline $\mathrm{SBP}(\mathrm{mmHg})$ & $141(5)^{\mathrm{a}, \mathrm{b}}$ & $130(4)^{\mathrm{c}}$ & $117(3)$ & 0.001 \\
\hline DBP (mmHg) & $78(2)^{b, c}$ & $76(3)$ & $71(2)$ & 0.079 \\
\hline Pulse Pressure $(\mathrm{mmHg})$ & $63(5)^{\mathrm{b}, \mathrm{c}}$ & $54(3)^{d}$ & $46(2)$ & 0.003 \\
\hline
\end{tabular}

Mean (standard error); ${ }^{\mathrm{a}} p<0.001$ vs control subjects; ${ }^{\mathrm{b}} p=$ NS vs DM MA-; ${ }^{\mathrm{c}} p<0.05$ vs controls; ${ }^{\mathrm{d}} p=\mathrm{NS}$ vs controls. BMI, body mass index; HRT, hormone replacement therapy; SBP, systolic blood pressure; DBP, diastolic blood pressure; NS, not significant.

ducer was placed 5 to $10 \mathrm{~cm}$ proximal to the anti-cubital crease and fixed in position by a stereotactic clamp. After good images were obtained, the edge-to-lumen interface was further optimised using depth and gain controls and an edge enhancement function. Images were recorded on s-VHS videotape (Sony MQSE 180) for retrospective analysis. A pneumatic tourniquet was placed around the left forearm, and after scanning the baseline artery diameter for one min the cuff was rapidly inflated to $50 \mathrm{mmHg}$ above systolic blood pressure for 5 min. Release of the cuff induced reactive hyperaemia and scanning was continued for five min longer to assess flow-mediated dilatation (FMD). Doppler flow velocity and flow rate ( $\mathrm{ml} / \mathrm{min})$ were calculated during baseline scanning and during the first $15 \mathrm{~s}$ of reactive hyperaemia. A second resting scan was obtained at least ten min after cuff deflation to ensure that the brachial artery diameter returned to the basal level before glyceryl trinitrate (GTN) treatment. Glyceryl trinitrate $400 \mu \mathrm{g}$ was sprayed sublingually and the artery was subsequently scanned for a further 6 min to assess GTNMD.

Analysis of FMD and GTNMD of the brachial artery was carried out using semi-automated edge detection software recently developed and validated within our department. During s-VHS playback into a digital frame-grabber, images were digitised into a personal computer. A rectangular region of interest (ROI) was drawn around the most representative section of the artery. A second ROI was selected around the ECG tracings, and a third ROI to calibrate diameter measurements. The computerised edge detection and wall tracking software system then automatically calculated brachial artery diameter, corresponding to the internal diameter and gated to the $\mathrm{R}$ wave of the ECG, with measurements taken at end-diastole (Fig. 1A). An experienced observer, blinded to the clinical state of the patients, did the analyses.

Arterial diameter estimates, corresponding to approximately 300 individual diameter measurements at each $\mathrm{R}$ wave, were used to calculate baseline diameters and maximal FMD and GTN responses. In the software, a third order polynomial function was applied to the FMD and GTNMD of the brachial artery to derive responses relative to the mean baseline diameter (Fig.1B). Responses are calculated as the percentage change in brachial artery diameter from baseline. The analytical (intra-observer) coefficient of variation of our computerised technique is in the order of $6.8 \%$ compared with $24.8 \%$ using more conventional visual estimation using ultra- sonic callipers. The resolving power of the method tested on phantom "arteries" is $8.7 \mu \mathrm{m}$. To test the reproducibility of the technique, ultrasound scans were carried out twice on 14 subjects within 1 week and analysed by an experienced observer. The mean (standard deviation) difference in brachial FMD was $1.5(0.8) \%$.

Statistical Methods. Results are expressed as mean (standard error). Group differences were analysed using analysis of variance (ANOVA) with Bonferroni adjustments for multiple comparisons (SPSS version 10.0, Statistical Package for Social Sciences, SPSS Inc., Chicago, Ill., USA). Skewed data (AER, $\mathrm{ACR}, \mathrm{HbA}_{1 \mathrm{c}}, \mathrm{Lp}(\mathrm{a})$ and serum triglyceride) were expressed as geometric mean (95\% confidence interval) and were log transformed before ANOVA analysis. Categorical variables were compared using the Chi-squared test. Because FMD and GTNMD are dependent on baseline arterial diameter, age and sex, the latter were entered as covariates in the analysis of variance model used to test group differences in FMD and GTNMD. Associations between FMD or GTNMD and independent variables were tested using univariate and multivariate regression analysis in both a pooled group analysis and in the diabetic patients alone. Statistical significance was taken as a $p$ value of less than 0.05 .

\section{Results}

The clinical characteristics of the 3 groups studied are shown in Table 1 . The volunteers were middle-aged with similar BMI and the group had a greater proportion of men than women. Diabetic patients had a long duration of diabetes and a similar daily dose of insulin. Diabetic patients with microalbuminuria (DM $\mathrm{MA}+$ ) had a significantly higher prevalence of hypertension, retinopathy and neuropathy. All the DM MA + subjects were on an ACE-inhibitor (enalapril 3 , captopril 3, and perindopril 11). Both diabetic groups had significantly higher systolic blood pressure and pulse pressure than the non-diabetic control group $(p=0.001)$. The DM MA + group had a signif- 
Table 2. Biochemical characteristics of the diabetic patients with (DMMA + ) and without (DMMA-) microalbuminuria and the non-diabetic control subjects.

\begin{tabular}{|c|c|c|c|c|}
\hline & DM MA + & DM MA- & Control subjects & $p$-value \\
\hline Fasting plasma glucose (mmol/l) & $11.2(1.2)^{\mathrm{a}, \mathrm{b}}$ & $10.7(1.1)^{b}$ & $4.8(0.1)$ & $<0.001$ \\
\hline Blood glucose during ultrasound test ( $\mathrm{mmol} / \mathrm{l})$ & $6.2(1.3)^{a, b}$ & $5.9(1.7)^{c}$ & $4.8(0.4)$ & 0.003 \\
\hline Glycated haemoglobin, $\mathrm{HbA}_{1}(\%)$ & $8.5^{\mathrm{a}, \mathrm{b}}(7.9-9.2)$ & $8.7^{\mathrm{b}}(8.1-9.5)$ & $5.1(4.2-6.0)$ & $<0.001$ \\
\hline $\mathrm{GFR}(\mathrm{ml} / \mathrm{min})$ & $84.9(3.6)^{\mathrm{d}, \mathrm{e}}$ & $114.9(7.3)^{\mathrm{c}}$ & $90.2(5.8)$ & 0.001 \\
\hline Urinary AER $(\mu \mathrm{g} / \mathrm{min})^{1}$ & $74.9^{\mathrm{d}}(63.0-137.2)$ & $3.6(2.9-6.0)$ & - & $<0.001$ \\
\hline Urinary ACR $(\mathrm{mg} / \mathrm{mmol})^{1}$ & $8.3^{\mathrm{b}, \mathrm{d}}(6.8-18.5)$ & $0.5^{\mathrm{e}}(0.4-0.8)$ & $0.5(0.3-0.7)$ & $<0.001$ \\
\hline LDL-cholesterol (mmol/l) & $3.4(0.2)$ & $3.2(0.2)$ & $2.9(0.2)$ & 0.171 \\
\hline Triglyceride $(\mathrm{mmol} / \mathrm{l})^{1}$ & $1.2(0.8-2.1)$ & $0.9(0.7-1.4)$ & $0.9(0.8-1.5)$ & 0.271 \\
\hline ApoB $(g / 1)$ & $1.0(0.1)$ & $1.0(0.1)$ & $0.9(0.1)$ & 0.247 \\
\hline $\operatorname{Lp}(\mathrm{a})(\mathrm{g} / \mathrm{l})^{1}$ & $0.1(0.1-0.4)$ & $0.1(0.1-0.2)$ & $0.1(0.1-0.2)$ & 0.448 \\
\hline Homocysteine $(\mu \mathrm{mol} / \mathrm{l})$ & $8.5(0.8)$ & $7.9(0.4)$ & $9.9(0.7)$ & 0.087 \\
\hline vWF (\%) & $89.5(7.8)$ & $90.0(9.4)$ & $88.5(13.4)$ & 0.762 \\
\hline
\end{tabular}

Mean (standard error) except ${ }^{1}$ geometric mean ( $95 \%$ confidence interval); ${ }^{\mathrm{a}} p=\mathrm{NS}$ vs DM MA-; ${ }^{\mathrm{b}} p<0.001$ vs controls; ${ }^{\mathrm{c}} p<0.01$ vs control subjects; ${ }^{\mathrm{d}} p<0.001$ vs DM MA-; ${ }^{\mathrm{e}} p=\mathrm{NS}$ vs control subjects;
Abbreviations: AER albumin excretion rate; ACR albumin: creatinine ratio; HDL high-density lipoprotein; LDL low-density lipoprotein; ApoB apolipoprotein B; Lp(a) lipoprotein (a); vWF von Willebrand factor

Table 3. Brachial artery vascular function in the diabetic patients with (DMMA + ) and without (DMMA-) microalbuminuria and the non-diabetic control subjects

\begin{tabular}{|c|c|c|c|c|}
\hline & $\mathrm{DM} \mathrm{MA}+$ & DM MA- & Controls & $p$-value \\
\hline Baseline diameter $(\mathrm{mm})$ & $3.9(0.1)$ & $3.6(0.1)$ & $3.5(0.1)$ & 0.079 \\
\hline Maximal post-ischaemic diameter ( $\mathrm{mm})$ & $4.0(0.1)$ & $3.8(0.1)$ & $3.8(0.1)$ & 0.265 \\
\hline Post-ischaemic FMD (\%) & $3.2(0.3)^{\mathrm{a}, \mathrm{b}}$ & $5.4(0.5)^{\mathrm{c}}$ & $7.9(0.6)$ & $<0.001$ \\
\hline Maximal diameter post-GTN (mm) & $4.4(0.1)$ & $4.2(0.1)$ & $4.3(0.1)$ & 0.761 \\
\hline GTNMD (\%) & $11.9(1.1)^{\mathrm{c}, \mathrm{d}}$ & $16.7(2.0)^{\mathrm{e}}$ & $20.0(1.2)$ & 0.001 \\
\hline
\end{tabular}

Mean (standard error); ${ }^{\mathrm{a}} p<0.01$ vs DM MA-; ${ }^{\mathrm{b}} p<0.001$ vs control subjects; ${ }^{\mathrm{c}} p=0.01$ vs controls; ${ }^{\mathrm{d}} p=\mathrm{NS}$ vs DM MA-; ${ }^{\mathrm{e}} p=\mathrm{NS}$ vs controls. Abbreviations: FMD flow-mediated dilatation; GTN glyceryl trinitrate; GTNMD glyceryl trinitrate-mediated dilatation.

icantly higher diastolic blood pressure than the control subjects $(p<0.05)$. The differences in blood pressure between the diabetic groups were not statistically significant.

The biochemical characteristics of the 3 groups are shown in Table 2. Fasting plasma glucose and $\mathrm{HbA}_{1 \mathrm{c}}$ were significantly higher in both diabetic groups than in the control group $(p<0.001)$. During ultrasound, although blood glucose was maintained between 3.5 and $8 \mathrm{mmol} / \mathrm{l}$, the mean blood glucose remained significantly higher than the fasting value for the control group $(p=0.003)$. Fasting plasma glucose, blood glucose during ultrasonography and $\mathrm{HbA}_{1 \mathrm{c}}$ did not differ significantly between the diabetic groups. The non-microalbuminuric diabetic patients (DM MA-) had a significantly lower serum creatinine $(p<0.001)$ than both control subjects and microalbuminuric patients (DMMA + ), with a corresponding significantly higher calculated GFR $(p=0.001)$. Albumin excretion rate (AER) was significantly higher in the DM MA + group than in the DM MA-group $(p<0.001)$. The albumin to creatinine ratio (ACR) was not significantly different between DM MAand control groups, but was significantly higher in the DMMA + group $(p<0.001)$. Lipids, lipoproteins, apolipoproteins and vonWillebrand factor (vWF) did not differ significantly among the groups. There was, however, a trend towards a significantly lower homocysteine in the DMMA- group $(p=0.087)$ and a trend to a higher fibrinogen in the DMMA + group $(p=0.097)$.

The results of the brachial artery vascular function are summarised in Table 3. Although baseline brachial artery diameter did not differ significantly among the groups, there was a trend to a significantly higher baseline diameter in microalbuminuric diabetic patients $(p=0.079)$. After adjusting for baseline arterial diameter, blood glucose during ultrasound, age and sex using analysis of covariance, FMD was significantly lower in the microalbuminuric (DM $\mathrm{MA}+)$ and non-microalbuminuric diabetic subjects (DM MA-) than in the control subjects $(p<0.001)$. 


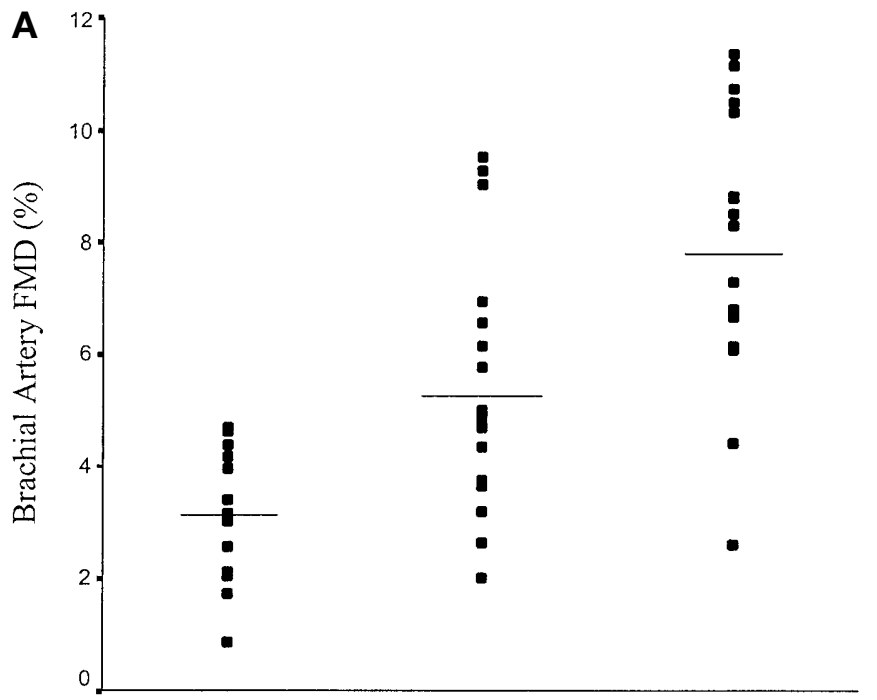

DMMA $+\quad$ DMMA - Control subjects

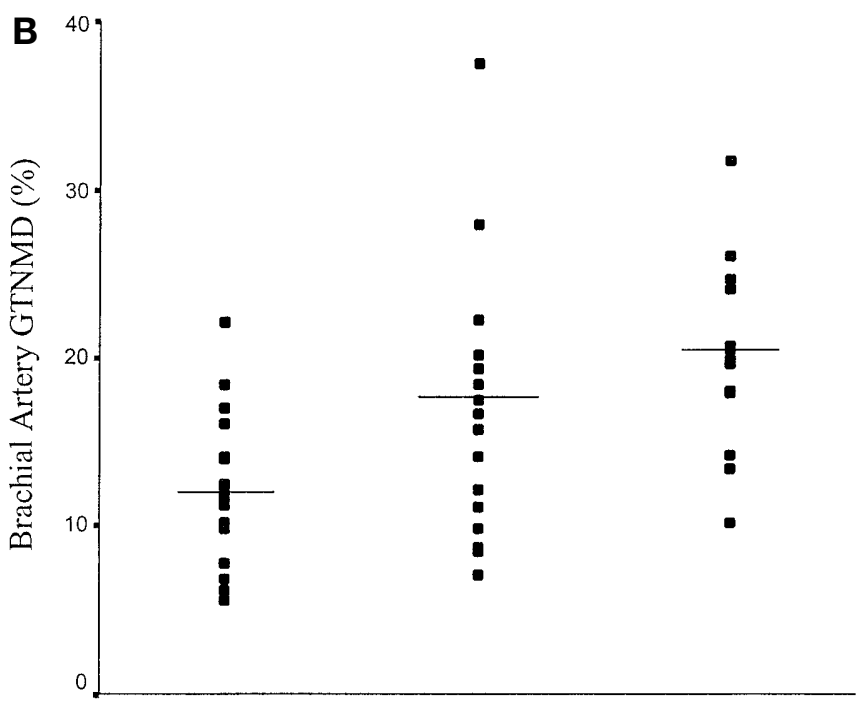

$\mathrm{DMMA}+$

DM MA -

Control subjects

Fig. 2A, B. Brachial artery vascular function in the diabetic patients with (DMMA + ) and without (DMMA-) microalbuminuria and the non-diabetic control subjects: (A) Post-ischaemic flow-mediated dilatation (FMD); (B) Glyceryl trinitratemediated dilatation (GTNMD).

Flow-mediated dilation was also significantly lower in the DM MA + subjects compared with the DM MA- subjects $(p<0.01)$. Resting blood flow (DM MA $-106 \pm 12 \mathrm{ml} / \mathrm{min}, \mathrm{DMMA}+136 \pm 11 \mathrm{ml} / \mathrm{min}$, $p=0.063)$ and percentage increase in blood flow during reactive hyperaemia (DMMA- $524 \pm 79 \%$, DMMA $+519 \pm 37 \%, p=0.960)$ was not different between the diabetic groups. GTNMD did differ significantly among groups $(p=0.001)$. After post-hoc Bonferroni adjustment, however, GTNMD was sig-
Table 4. Multiple regression models showing association between post-ischaemic FMD of the brachial artery and albumin excretion rate and $\mathrm{HbA}_{1 \mathrm{c}}$; glomerular filtration rate and $\mathrm{HbA}_{1 \mathrm{c}}$ adjusted for LDL-cholesterol, systolic blood pressure, disease duration, blood glucose during ultrasound and brachial artery diameter

\begin{tabular}{|c|c|c|}
\hline Predictor Variable & $\begin{array}{l}\text { Regression } \\
\text { coefficient } \\
\text { (standard error) }\end{array}$ & $p$-value \\
\hline Albumin excretion rate $(\mu \mathrm{g} / \mathrm{min})$ & $-0.547(0.201)$ & 0.012 \\
\hline $\mathrm{HbA}_{1 \mathrm{c}}(\%)$ & $-0.510(0.244)$ & 0.047 \\
\hline Systolic blood pressure $(\mathrm{mmHg})$ & $0.034(0.018)$ & 0.075 \\
\hline $\begin{array}{l}\text { Blood glucose during ultrasound } \\
(\mathrm{mmol} / \mathrm{L})\end{array}$ & $-1.964(1.481)$ & 0.197 \\
\hline LDL-cholesterol (mmol/L) & $1.375(1.435)$ & 0.347 \\
\hline Duration of diabetes (yr) & $-0.012(0.032)$ & 0.710 \\
\hline Brachial artery diameter $(\mathrm{mm})$ & $-8.094(2.208)$ & 0.001 \\
\hline \multicolumn{3}{|l|}{ Adjusted $\mathrm{R}^{2}=0.53$} \\
\hline Predictor Variable & $\begin{array}{l}\text { Regression } \\
\text { coefficient } \\
\text { (standard error) }\end{array}$ & $p$-value \\
\hline Glomerular filtration rate $(\mathrm{ml} / \mathrm{min})$ & $0.032(0.012)$ & 0.014 \\
\hline $\mathrm{HbA}_{1 \mathrm{c}}(\%)$ & $-0.589(0.249)$ & 0.026 \\
\hline Systolic blood pressure $(\mathrm{mmHg})$ & $0.026(0.017)$ & 0.150 \\
\hline $\begin{array}{l}\text { Plasma glucose during ultrasound } \\
(\mathrm{mmol} / \mathrm{L})\end{array}$ & $-2.530(1.489)$ & 0.102 \\
\hline LDL-cholesterol (mmol/L) & $0.647(1.439)$ & 0.657 \\
\hline Duration of diabetes (yr) & $-0.009(0.033)$ & 0.775 \\
\hline Brachial artery diameter $(\mathrm{mm})$ & $-7.788(2.258)$ & 0.002 \\
\hline
\end{tabular}

Adjusted $\mathrm{R}^{2}=0.53$

nificantly lower only in the DM MA + subjects compared with control subjects $(p=0.01)$, whereas GTNMD between DM MA- and the control subjects did not differ significantly. The ratio of FMD to GTNMD was also significantly lower in the DM MA + subjects than in the control subjects $(0.29 \pm 0.13$ vs $0.40 \pm 0.12, p=0.013)$. The individual values for FMD and GTNMD in the 3 groups are shown in Figure 2.

In multiple regression analysis using data pooled from all groups, brachial artery diameter (regression coefficient, $r=7.40 \pm 2.04, p=0.001$ ), the presence of diabetes $(r=2.24 \pm 0.73, p=0.004)$ and urinary albumin to creatinine ratio $(r=-0.47 \pm 0.22, p=0.036)$ were significant independent predictors of FMD. Within the diabetic group alone, univariate regression analysis showed that FMD was inversely correlated with brachial artery diameter $(r=-0.50$, $p=0.001)$ and AER $(r=-0.48, p=0.002)$ and directly correlated with GFR $(r=0.46, p=0.004)$. No significant correlations were found with other variables, in particular LDL-cholesterol, $\mathrm{HbA}_{1 \mathrm{c}}$, blood glucose during ultrasound or blood pressure. Because GFR was strongly correlated with AER $(r=-0.52$, $p=0.001)$, they were entered into separate multiple 
regression models. In multiple regression analyses adjusting for brachial artery diameter, increasing AER (or decreasing GFR) and $\mathrm{HbA}_{1 \mathrm{c}}$ were significantly and independently associated with impaired FMD (Table 4). Using univariate regression, GTNMD in the diabetic groups was inversely correlated with brachial artery diameter $(r=-0.47$, $p=0.004)$ and disease duration $(r=-0.29, p=0.050)$ only. In separate multiple regression analyses after adjusting for brachial artery diameter GTNMD was not significantly correlated with any of the predictor variables (LDL-cholesterol, systolic blood pressure, disease duration, $\mathrm{HbA}_{1 \mathrm{c}}$, blood glucose during ultrasound, AER or GFR).

\section{Discussion}

This study examined conduit artery endothelial function in diabetic patients at near-normoglycaemia using a euglycaemic insulin clamp and using computerised edge-detection software. We show that Type I diabetic patients have endothelial dysfunction compared with the control subjects matched for age and sex, an abnormality mostly marked in those with microalbuminuria, despite treating with ACE inhibitors. Endothelial function in diabetic patients was negatively correlated with microalbuminuria and glycaemic control, independent of blood pressure and LDL-cholesterol. Patients with microalbuminuria and Type I diabetes also had impaired glyceryl trinitrate mediated vasodilatation.

While several studies have measured conduit artery endothelial function in Type I diabetes, there have been conflicting results $[10-15,18]$. Confounding factors include variations in blood glucose concentrations [16,17], gender differences, [19] and poor reproducibility of standard ultrasound procedure [20]. In our study, diabetic patients were studied at near-normoglycaemia. Although blood glucose in both diabetic groups remained significantly higher than in the healthy control group during ultrasound, it was in the target range of 3.5 to $8.0 \mathrm{mmol} / \mathrm{l}$; hyperglycaemia associated with endothelial dysfunction has only been documented at glucose concentrations exceeding $8.0 \mathrm{mmol} / \mathrm{l}[16,17]$. Furthermore, blood glucose during ultrasound was not significantly correlated with endothelial function. To increase methodological precision, we used a computerised edge-detection software program, which improves our analytical coefficient of variation from approximately $25 \%$ to $6.8 \%$. Finally, the difference in conduit artery endothelial function between the two diabetic groups and between the diabetic groups and the healthy control group persisted after adjusting for age and sex.

In agreement with other studies [11-13], we have shown that normoalbuminuric Type I diabetic patients also have endothelial dysfunction compared with healthy control subjects, suggesting that endothelial dysfunction could precede microalbuminuria as a cardiovascular risk factor. Also consistent with other data [2], microalbuminuric Type I diabetic patients had worse endothelial dysfunction and microalbuminuria correlated with impaired endothelial function. Contributory mechanisms include increased transcapillary escape rate of albumin [21] and insulin resistance [22] which has been shown to be associated with microalbuminuria independent of glycaemic control [23, 24]. Lipoprotein abnormalities such as low HDL- and increased LDL-cholesterol are more common in microalbuminria [25-27], and can induce endothelial dysfunction [28, 29]. We did not, however, find a difference in plasma lipids and lipoproteins between the diabetic groups. Two possible reasons for the discrepancy in previous reports are that our patients had better long-term glycaemic control [30] and less albuminuria $[25,31]$. We also found no difference in $\mathrm{Lp}(\mathrm{a})$ concentrations between the diabetic groups, but whether $\mathrm{Lp}(\mathrm{a})$ is increased in Type I diabetic patients with microalbuminuria remains contentious [32-34]. Finally, in patients with microalbuminuria the higher mean arterial and pulse pressures, which reflect reduced arterial compliance, might have contributed to endothelial dysfunction [35].

A further finding in our study was that patients with micoralbuminuric Type I diabetes had endothelial dysfunction despite being on ACE-inhibitor therapy. The effect of ACE-inhibitors on endothelial function in diabetic and non-diabetic subjects is not clear [36-39]. Improvement in endothelial function with ACE-inhibitors can depend on the vascular bed studied, the tissue affinity of the ACE-inhibitor used and the ACE genotype [36, 38, 40]. Our microalbuminuric diabetic patients were on ACE-inhibitors with low tissue affinity, which could explain the apparent lack of effect on endothelial function. Their FMD, however, could have been worse off ACE-inhibitors. Furthermore, our blood pressure data suggest that our microalbuminuric patients might have been on sub-therapeutic doses of ACE-inhibitors.

Glycaemic control, as measured by $\mathrm{HbA}_{1 \mathrm{c}}$, was also found to be a significant, independent predictor of endothelial dysfunction. Contributory mechanisms include increased oxidative stress as a result of increased generation of free radicals due to hyperglycaemia [41]. Furthermore, hyperglycaemia induces protein kinase $\mathrm{C}$ activation and depletion of essential nitric oxide synthase cofactors resulting in deactivation of nitric oxide and nitric oxide synthase, thereby reducing nitric oxide bioavailability [42, 43]. Hyperglycaemia has also recently been shown to enhance expression of endothelin-1, a vasoconstrictor that antagonizes the effects of nitric oxide [44]. Finally, advanced glycation endproducts (AGE) [45] can directly quench nitric oxide produced by endothelial cells [46]. 
Calculated GFR was significantly lower in the microalbuminuric diabetic group, suggesting subclinical GFR impairment and was negatively correlated with microalbuminuria. The correlation between GFR and endothelial function is likely to be a function of the strong association between albumin excretion rate and GFR, particularly because serum creatinine was not impaired. Endothelial dysfunction could, however, also be mediated by increased circulating concentrations of nitric oxide synthase inhibitors in renal impairment, such as asymmetric dimethyl arginine [47].

GTNMD was impaired only in the microalbuminuric diabetic patients. Possible mechanisms involved would include nitric oxide deactivation by free radicals and AGE deposition as a consequence of chronic hyperglycaemia [22, 46]. Deposition of AGE is a chronic event and could explain the weak correlation between duration of diabetes and impaired GTN responses. The FMD:GTNMD ratio was also impaired in microalbuminuric patients, suggesting that the impaired FMD response was out of proportion to the impaired GTN response.

Study limitations. It is not likely that vasodilatory effects of administered insulin could have confounded our results, as these effects, mainly seen in the microcirculation, result in augmented rather than suppressed nitric oxide-mediated flow responses [48]. A further limitation is the potential confounding effect on endothelial function of the diversity in age and sex in the study groups. However, we case-matched for these factors, so that group differences in FMD remained significant after adjusting for age and sex in statistical analyses. A formal study of the effect of sex on FMD in our diabetic groups would require a larger sample size.

Implications for clinical practice and future studies. That endothelial dysfunction precedes microalbuminuria in Type I diabetes in our study suggests that it could be an earlier risk marker for cardiovascular disease and a target for therapy. Association with $\mathrm{HbA}_{1 \mathrm{c}}$ reinforces the need to improve glycaemic control [49]. The roles of anti-oxidants and inhibitors of AGE formation in Type I diabetes merit further study given the link between endothelial dysfunction, hyperglycaemia and oxidative stress. It is also possible that insulin resistance associated with microalbuminuria might have contributed to endothelial dysfunction and the benefits of insulin sensitisers need to be evaluated in these patients.

In conclusion, we have shown that endothelial function is impaired in patients with Type I diabetes mellitus in the absence of acute hyperglycaemia and this could reflect increased cardiovascular risk before the onset of microalbuminuria. Measurement of endothelial function of the brachial artery could be use- ful in identifying a high-risk group of diabetic patients who might benefit from more intensive conventional and new therapies.

Acknowledgements. We acknowledge the financial support from the National Health and Medical Research Council and the Medical Research Foundation of Royal Perth Hospital. We are grateful to Enid Pereira and Mary Ann Powell for their assistance with monitoring patients while on insulin infusions and to Diabetes Physicians at Royal Perth Hospital for referring patients for the study.

\section{References}

1. Borch-Johnsen K, Kreiner S (1989) Proteinuria: value as predictor of cardiovascular mortality in insulin dependent diabetes mellitus. BMJ; 294: 1651-1654

2. Deckert T, Kofoed-Enevoldsen A, Norgaard K, BorchJohnsen K, Feldt-Rasmussen B, Jensen T (1992) Microalbuminuria. Implications for micro- and macrovascular disease. Diabetes Care 15: 1181-1191

3. Rubanyi GM (1993) The role of endothelium in cardiovascular homeostasis and diseases. J Cardiovasc Pharmacol 22 [Suppl 4]: S1-S14

4. Celermajer DS, Sorensen KE, Gooch VM et al. (1992) Non-invasive detection of endothelial dysfunction in children and adults at risk of atherosclerosis. Lancet 340: 1111-1115

5. Anderson TJ, Uehata A, Gerhard MD et al. (1995) Close relation of endothelial function in the human coronary and peripheral circulations. J Am Coll Cardiol 26: 1235-1241

6. Neunteufl T, Katzenschlager R, Hassan A et al. (1997) Systemic endothelial dysfunction is related to the extent and severity of coronary artery disease. Atherosclerosis 129: 111-118

7. Suwaidi JA, Hamasaki S, Higano ST, Nishimura RA, Holmes DR Jr, Lerman A (2000) Long-term follow-up of patients with mild coronary artery disease and endothelial dysfunction. Circulation 101: 948-954

8. Neunteufl T, Heher S, Katzenschlager R et al. (2000) Late prognostic value of flow-mediated dilation in the brachial artery of patients with chest pain. Am J Cardiol 86: 207-210

9. Schachinger V, Britten MB, Zeiher AM (2000) Prognostic impact of coronary vasodilator dysfunction on adverse long- term outcome of coronary heart disease. Circulation 101: 1899-1906

10. Clarkson P, Celermajer DS, Donald AE et al. (1996) Impaired vascular reactivity in insulin-dependent diabetes mellitus is related to disease duration and low density lipoprotein cholesterol levels. J Am Coll Cardiol 28: 573-579

11. Meeking DR, Cummings MH, Thorne S et al. (1999) Endothelial dysfunction in Type I diabetic subjects with and without microalbuminuria. Diabet Med 16: 841-847

12. Zenere BM, Arcaro G, Saggiani F, Rossi L, Muggeo M, Lechi A (1995) Noninvasive detection of functional alterations of the arterial wall in IDDM patients with and without microalbuminuria. Diabetes Care 18: 975-982

13. Lekakis J, Papamichael C, Anastasiou H et al. (1997) Endothelial dysfunction of conduit arteries in insulin-dependent diabetes mellitus without microalbuminuria. Cardiovasc Res 34: 164-168

14. Lambert J, Aarsen M, Donker AJ, Stehouwer CD (1996) Endothelium-dependent and -independent vasodilation of 
large arteries in normoalbuminuric insulin-dependent diabetes mellitus. Arterioscler Thromb Vasc Biol 16: 705-711

15. Enderle MD, Benda N, Schmuelling RM, Haering HU, Pfohl M (1998) Preserved endothelial function in IDDM patients, but not in NIDDM patients, compared with healthy subjects. Diabetes Care 21(2): 271-277

16. Williams SB, Goldfine AB, Timimi FK et al. (1998) Acute hyperglycemia attenuates endothelium-dependent vasodilation in humans in vivo. Circulation 1998; 97: 1695-1701

17. Kawano H, Motoyama T, Hirashima O et al. (1999) Hyperglycemia rapidly suppresses flow-mediated endotheliumdependent vasodilation of brachial artery. J Am Coll Cardiol 34: 146-154

18. Pinkney JH, Downs L, Hopton M, Mackness MI, Bolton CH (1999) Endothelial dysfunction in Type I diabetes mellitus: relationship with LDL oxidation and the effects of vitamin E. Diabet Med 16: 993-999

19. Chan NN, Vallance P, Colhoun HM (2000) Nitric oxide and vascular responses in Type I diabetes. Diabetologia 43: 137-147

20. Hardie KL, Kinlay S, Hardy DB, Wlodarczyk J, Silberberg JS, Fletcher PJ (1997) Reproducibility of brachial ultrasonography and flow-mediated dilatation (FMD) for assessing endothelial function. Aust N Z J Med 27: 649-652

21. Norgaard K, Jensen T, Feldt-Rasmussen B (1993) Transcapillary escape rate of albumin in hypertensive patients with Type I (insulin-dependent) diabetes mellitus. Diabetologia 36: 57-61

22. Playford DA, Watts GF (1999) Endothelial dysfunction, insulin resistance and diabetes: exploring the web of causality. Aust N Z J Med 29: 523-534

23. Yip J, Mattock MB, Morocutti A, Sethi M, Trevisan R, Viberti G (1993) Insulin resistance in insulin-dependent diabetic patients with microalbuminuria. Lancet 342: 883-887

24. Makimattila S, Virkamaki A, Groop PH et al. (1996) Chronic hyperglycemia impairs endothelial function and insulin sensitivity via different mechanisms in insulin-dependent diabetes mellitus. Circulation 94: 1276-1282

25. Vannini P, Ciavarella A, Flammini M et al. (1984) Lipid abnormalities in insulin-dependent diabetic patients with albuminuria. Diabetes Care 7: 151-154

26. Jensen T, Stender S, Deckert T (1988) Abnormalities in plasmas concentrations of lipoproteins and fibrinogen in Type I (insulin-dependent) diabetic patients with increased urinary albumin excretion. Diabetologia 31: 142-145

27. Winocour PH, Durrington PN, Bhatnagar D, Ishola M, Mackness M, Arrol S (1991) Influence of early diabetic nephropathy on very low density lipoprotein (VLDL), intermediate density lipoprotein (IDL), and low density lipoprotein (LDL) composition. Atherosclerosis 89: 49-57

28. O'Brien SF, Watts GF, Playford DA, Burke V, O'Neal DN, Best JD (1997) Low-density lipoprotein size, high-density lipoprotein concentration, and endothelial dysfunction in non-insulin-dependent diabetes. Diabet Med 14: 974-978

29. Treasure CB, Klein JL, Weintraub WS et al. (1995)Beneficial effects of cholesterol-lowering therapy on the coronary endothelium in patients with coronary artery disease. N Engl J Med 332: 481-487

30. Watts GF, Naumova R, Slavin BM et al. (1989) Serum lipids and lipoproteins in insulin-dependent diabetic patients with persistent microalbuminuria. Diabet Med 6: 25-30

31. Winocour PH, Durrington PN, Ishola M, Anderson DC, Cohen H (1997) Influence of proteinuria on vascular disease, blood pressure, and lipoproteins in insulin dependent diabetes mellitus. BMJ 294: 1648-1651

32. Schernthaner G, Kostner GM, Dieplinger H, Prager R, Muhlhauser I (1983) Apolipoproteins (A-I, A-II, B),
Lp(a) lipoprotein and lecithin: cholesterol acyltransferase activity in diabetes mellitus. Atherosclerosis 49: 277-293

33. Irish AB, Simons LA, Simons J (1992) Lipoprotein(a) concentration in diabetes: relationship to proteinuria and diabetes control. Aust N Z J Med 22: 329-333

34. Ritter MM, Loscar M, Richter WO, Schwandt P (1993) Lipoprotein(a) in diabetes mellitus. Clin Chim Acta 214: 45-54

35. Berry KL, Skyrme-Jones RA, Cameron JD, O'Brien RC, Meredith IT (1999) Systemic arterial compliance is reduced in young patients with IDDM. Am J Physiology 276: H1839-H1845

36. Anderson TJ, Elstein E, Haber H, Charbonneau F (2000) Comparative study of ACE-inhibition, angiotensin II antagonism, and calcium channel blockade on flow-mediated vasodilation in patients with coronary disease (BANFF study). J Am Coll Cardiol 35: 60-66

37. McFarlane R, McCredie RJ, Bonney MA et al. (1999) Angiotensin converting enzyme inhibition and arterial endothelial function in adults with Type I diabetes mellitus. Diabet Med 16(1): 62-66

38. O'Driscoll G, Green D, Rankin J, Stanton K, Taylor R (1999) Improvement in endothelial function by angiotensin converting enzyme inhibition in insulin-dependent diabetes mellitus. J Clin Invest 100: 678-684

39. Mancini GB, Henry GC, Macaya C et al. (1996) Angiotensin-converting enzyme inhibition with quinapril improves endothelial vasomotor dysfunction in patients with coronary artery disease. The TREND (Trial on Reversing ENdothelial Dysfunction) Study. Circulation 94: 258-265

40. Deedwania PC (2000) Endothelium: a new target for cardiovascular therapeutics. J Am Coll Cardiol 35: 67-70

41. Tesfamariam B, Cohen RA (1992) Free radicals mediate endothelial cell dysfunction caused by elevated glucose. Am J Physiol 263: H321-H326

42. Poston L, Taylor PD (1995) Endothelium-mediated vascular function in insulin-dependent diabetes mellitus. Clin Sci (Colch) 88: 245-255

43. Hawthorne GC, Bartlett K, Hetherington CS, Alberti KG (1989) The effect of high glucose on polyol pathway activity and myoinositol metabolism in cultured human endothelial cells. Diabetologia 32: 163-166

44. Park JY, Takahara N, Gabriele A et al. (2000) Induction of endothelin-1 expression by glucose: an effect of protein kinase $C$ activation. Diabetes 49: 1239-1248

45. Brownlee M, Cerami A, Vlassara H (1988) Advanced glycosylation end products in tissue and the biochemical basis of diabetic complications. N Engl J Med 318: 1315-1321

46. Bucala R, Tracey KJ, Cerami A (1991) Advanced glycosylation products quench nitric oxide and mediate defective endothelium-dependent vasodilatation in experimental diabetes. J Clin Invest 87: 432-438

47. Vallance P, Leone A, Calver A, Collier J, Moncada S (1992) Accumulation of an endogenous inhibitor of nitric oxide synthesis in chronic renal failure. Lancet 339: 572-575

48. Taddei S, Virdis A, Mattei P, Natali A, Ferrannini E, Salvetti A (1995) Effect of insulin on acetylcholine-induced vasodilation in normotensive subjects and patients with essential hypertension. Circulation 92: 2911-2918

49. The Diabetes Control and Complications Trial Research Group (1993) The effect of intensive treatment of diabetes on the development and progression of long-term complications in insulin-dependent diabetes mellitus. N Engl J Med 329: 977-986 